\title{
Global Approach to Neuroendocrine Tumors Neoplasms
}

Maria del Carmen Pantín González*, Diego Malón Giménez, Beatriz Antón Pascual, Beatriz Losada Vila, Esther Llorente, Nadia Sánchez Baños, Manuela Martín, David Gutiérrez Abad and Juan Antonio Guerra

Oncology Department, University Hospital Fuenlabrada, Madrid, Spain

${ }^{\star}$ Corresponding author: Maria del Carmen Pantín González, Oncology Department, University Hospital, Fuenlabrada, Madrid, Spain, E-mail: carmenpantin1991@gmail.com Received: April 05, 2020; Accepted: April 15, 2020; Published: April 20, 2020

\section{Introduction}

The incidence of neuroendocrine tumors (TNE) is about 2.5-5 new cases per 100,000 inhabitants annually. They can appear in any location and $15 \%$ are diagnosed in the small bowel. TNEs can be functional with diarrhea, hot flashes, weight loss ... or not functionaries, that usually debut unspecifically with nausea, abdominal pain, anorexia intestinal obstruction and bleeding. This case reflects the evolution, monitoring and management of an intestinal TNE.

\section{History}

A 55-year-old male patient without medical illnesses to be highlighted consulted in June 2018 for 3-4 daily episodes of watery diarrhea, dizziness and loss of 5 kilos in the last two months.

\section{Physical Examination}

Constants: Blood pressure: 120/75 $\mathrm{mmHg}$, 98\% baseline saturation, afebrile.
Laterocervical, submandibular, supra or infraclavicular adenopathies are not palpated.

Pulmonary and cardiac auscultation was normal. The abdomen wasn `t pain and without megalias or masses.

\section{Supplementary Tests}

- Analytical with renal function, ions, liver profile and hemograme within normal.

- Basal chromogranin $159.5 \mathrm{ng} / \mathrm{Ml}(<84.7 \mathrm{ng} / \mathrm{ml})$, Urinary 5-hydroxyindoleacetic acid (5-HIAA): $21 \mu \mathrm{mol} / \mathrm{d}(0-14 \mu \mathrm{mol} / \mathrm{d})$

- Thoracoabdominopelvic CT scan showed a carcinoid tumor located in jejunal loops, liver metastases for all segments (the largest is $26 \mathrm{~mm}$ ) and is located in the liver segment 4 . (Figure 1)

- Somatostatin receptor imaging: Liver metastases and jejunal tumor is confirmed.

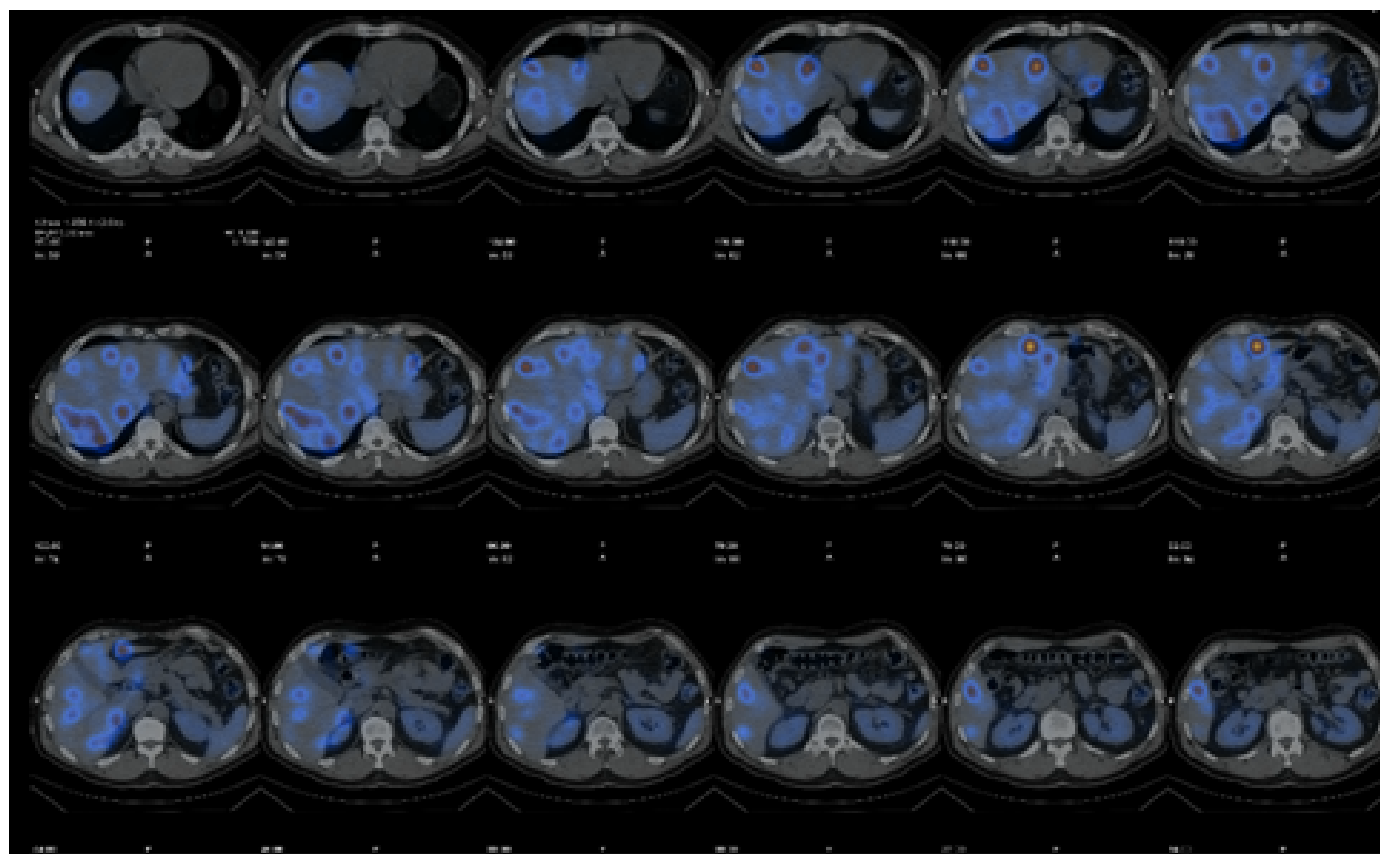

Figure 1. Somatostatin receptor imaging: liver metastases 
- Endoscopy: without injuries.

- Liver biopsy: metastasis of well-differentiated TNE GRADE 1 (WHO 2010). Ki-67 1-2\%. The Cells are positive for neuroendocrine markers such as chromogranin, synaptophysin, CD56.

\section{Diagnosis}

Neuroendocrine tumor well-differentiated ileo-jejunal Grade 1 (Ki-67 1-2\%) with metastatic involvement in all liver segments.

\section{Treatment}

The patient started treatment with lanreotide autogel $120 \mathrm{mg}$ every 28 days in October 2018 with improvement of the carcinoid syndrome. The primary tumor is intervened in January 2019 (small bowel resection: p T3, N1, LV0, Pn1 R0.) No possibility of liver resection due to extensive tumor involvement without the possibility of leaving enough hepatic remnants.

\section{Evolution}

After surgery, the study with serum chromogranin A and 5-HIAA was normal.

The patient continued with somatostatin analogues since January 2019 being completely asymptomatic and with an excellent quality of life. The patient followed clinical controls and radiological images every 6 months.

In November 2019, he went again to the emergency room for asthenia and watery diarrhea (4-5 daily episodes) the week prior to the administration of the analog. Markers were not elevated.

A thoracoabdominopelvic scan showed hepatic tumor progression with growth of all hepatic metastases. No other visceral or adenopathic lesions.

The somatostatin analogue was taken biweekly, so the symptoms disappeared again.

A new somatostatin receptor imaging showed positivity for liver metastases. Chromogranin A markers and 5-HIAA did not rise at any time.

Once the case has been assessed in the TNE committee, it is agreed to start treatment with radionuclides (Lutetium-177) 200 $\mathrm{mCi}$ in December 2019 and maintenance of somatostatin analogues (monthly dosage) between Lutetium dose.

The patient to date has received three doses of 177-LU-
DOTETATE with excellent tolerance, asymptomatic and without any documented adverse effects. At the present time, there is no evidence of disease progression.

\section{Discussion}

This case reflects the evolution and management of a jejunal TNE with hepatic metastatic at baseline which was considered unresectable.

In all TNE, the possibility of primary surgery should always be assessed even if there is metastatic disease since up to $30 \%$ of these tumors have liver involvement at diagnosis and survival at 5 years after primary resection is greater than $95 \%$ [1].

After surgery, it was decided to continue treatment with analogue because it was a stage IV with liver metastases that could not be resected.

The treatment controlled the symptoms during 13 months, according to the CLARINET study [2].

After confirming the progression, systematic treatment with radionuclides (PRRT) is decided. The PRRT consists of the systemic administration of the Ytrio-90 or Lutetium-177 conjugated radionuclides with a somatostatin analogue, through the acid chelating agent 1,4,7,10-tetraazacyclo-dodecane-1,4,7,10-tetraacetic acid (DOTA).

Radionuclide therapy has a phase III trial (NETTER-1) that compared treatment with 177Lu-DOTATATE every 4 weeks vs. LAR high dose octreotide, in patients with bowel TNE unresectable, with positive somatostatin receptors and with progression to analogs in the first line. Progression-free survival was 28.4 months versus 8 months in the control arm. (hazard ratio (HR) $0.21,95 \% \mathrm{CI}, 0.14-0.33$, p $<0.0001)$ [3].

In conclusion, PRRTs is indicated in patients with midgut TNEs well differentiated, metastatic, unresectable, in progression to somatostatin analogues and with positive somatostatin receptors.

\section{References}

1. Chan DL, Moody L, Segelov E, Metz D, Strosberg J, Pavlakis N, et al. (2018) Follow-up for resected gastroenteropancreatic neuroendocrine tumours (GEP-NETs): a practice survey of the Commonwealth Neuroendocrine collaboration (CommNETs) and North American Neuroendocrine Tumor Society (NANETS). Neuroendocrinology 107(1):32-41.

2. Caplin ME, Pavel M, Ćwikła JB, Phan AT, Raderer M, et al. (2014) Lanreotide in metastatic enteropancreatic neuroendocrine tumors. N Engl J Med 371:224-33.

3. Strosberg J, El-Haddad G, Wolin E, Hendifar A, Yao J, Chasen B, et al. (2017) Phase 3 trial of 177Lu-dotatate for midgut neuroendocrine tumors. N Engl J Med 376:125-35

\section{Citation:}

Pantín González MC, Giménez DM, Pascual BA, Vila BL, Llorente E, et al. (2020) Global Approach to Neuroendocrine Tumors Neoplasms Microbiol Immunol Pathol Volume 2(1): 1-2. 